\title{
Web and WSN'S Integration: The Web of Things (WoT)
}

\author{
Hathal Salamah A. Alwageed \\ Department of Computer Engineering and Network \\ Aljouf University, Saudi Arabia
}

\begin{abstract}
The novel trend of IPv6 over Low power Wireless Personal Area Networks (6LoWPAN) has enlivened the compromise of Wireless Sensor Networks (WSNs) and Smart objects with the ubiquitous Internet. Meanwhile, the Term CoAP stands for Constrained Application Protocol has made it possible to bestow resource obliged devices all along RESTful web services functionalities and thus to organize Wireless Sensor Networks and smart things or objects with the Web. The exercise of Web services on top of IP based Wireless Sensor Networks holds up the software reusability and reductions the capriciousness of the application change. This exertion spotlight RESTful Wireless Sensor Networks. It depicts CoAP, highlights the principal appears differently in relation to Hyper Text Transfer Protocol (HTTP) and reports the delayed consequences of a fundamental investigation exhibiting the benefits of CoAP in regards to compel usage diverged from Hyper Text Transfer Protocol (HTTP). We have depicted the arrangement and progression of an end-toend IP based auxiliary arranging (architecture) joining a CoAP more than 6LowPAN Contiki based Wireless Sensor Networks with a Hyper Text Transfer Protocol (HTTP) over IP based application. The application consents a customer to get to Wireless Sensor Networks data particularly from a Web program.
\end{abstract}

\section{Keywords}

WSN'S, WWW, WoT, CoAP

\section{INTRODUCTION}

Late Progression and maturity in the technology of Wireless Sensor Network (WSN) and the exploitation of the Internet Protocol (IP) in resource propelled/constrained contraptions (devices) has definitely changed the Internet scene. An outsized number of smart objects will be joined with the Internet to edge the assumed Internet of Things (IoT). The IoT will interface physical settings to the Internet, unleashing stimulating potential results and challenges for a blended sack of utilization regions, for instance, E-health, home and building automation, smart metering, smart grid [Atzori, L., et al]. The usage of IP advancement on embedded devices has been starting late progressed by the work of the IP for Smart Objects (IPSO) Alliance [ipso-alliance.org], a gathering of critical Information Technology and telecommunication players and remote silicon traders. Meanwhile, the Internet Engineering Task Force (IETF) has done liberal regulation development on IPv6 over Low power Wireless Personal Area Networks (6LoWPAN) [Kushalnagar]. This new-fangled standard engages the usage of IPv6 in Low-power and Lossy Networks (LLNs, for instance, those in light of the IEEE 802.15.4 standard [Shelby, 2009]. Despite 6LowPAN, IETF Routing over Low-power and Lossy frameworks (ROLL) Working Group has created and shown another IP routing protocol for internetworking of smart things. For [Vasseur, J. $P$ et al] Low-power and Lossy frameworks (RPL) [Vasseur, J. $\mathrm{P}$ et al] this protocol is called IPv6 Routing Protocol. One of the noteworthy focal points of IP based frameworks or networking in LLNs is to facilitate the usage of standard web services architectures devoid of bringing into play application entrances (gateways). Accordingly, smart things or objects won't simply be composed with the web also with the Web. This coordination is portrayed as the Web of Things (WoT). The advantage of the Web of Things is that smart things applications can be in light of top Representational State Transfer (REST) architectures. REST architectures license applications to rely on upon pretty nearly coupled services which can be granted and reused. In a REST building plan a benefit is a reflection controlled by the server and recognized by a Universal Resource Identifier (URI). The benefits are decoupled by the organizations and in this way resources can be subjectively addressed by strategy for diverse arrangements, for instance, JSON or XML. The benefits are gotten to and controlled by an application protocol considering client/server request/responses. REST is not altering to a particular application tradition. Regardless, most of REST architectures nowadays utilize Hypertext Transfer Protocol (HTTP). Hypertext Transfer Protocol controls resources by strategy for its schedules GET, POST, PUT, et cetera [6]. REST architectures grant Machine-to-Machine (M2M) and IoT applications to be created on top of web services which can be bestowed and reused. The sensors get the chance to be special resources perceived by Universal Resource Identifier's, addresses with self-decisive associations and controlled with the same frameworks/networks as Hypertext Transfer Protocol (HTTP). Therefore, RESTful WSNs drastically decrease the application change diserse quality. The usage of web services in LLNs is not clear as an aftereffect of the differentiations between Internet applications and IoT or M2M applications. IoT or M2M applications are brief and web services live in battery worked devices which as a general rule rest and wakeup exactly when there is data movement to be exchanged. Similarly, such applications oblige a multicast and odd correspondence appeared differently in relation to the unicast and synchronous approach of standard Internet applications [Trifa, V., et al]. The Internet Engineering Task Force (IETF) Constrained RESTful Environments (CoRE) Working Group has done critical systematization work for bringing the web services perfect model into smart objects frameworks/networks. The CoRE group has described a REST based web trade tradition called Constrained Application Protocol (CoAP). CoAP joins the HTTP functionalities which have been re-illustrated considering the low changing power and essentialness usage necessities of minimal introduced contraptions (devices), for instance, sensors. To make the protocol appropriate to M2M and IoT applications, diverse new functionalities have been incorporated [Shelby, Z., et al]. With 6LoWPAN developments getting the opportunity to be add to, the WoT has started accepting noteworthy part among the investigation bunch. Distinctive investigation articles putting forward REST/HTTP architectures for WSNs have starting late appeared. [D-Haggerty, S, et al] anticipates a RESTful 
development demonstrating which allows instruments and diverse producers of physical information to direct appropriate their data. [Kovatsch, M., et al], suggest a REST/HTTP framework/network for Home Automation. [Mayer, S., et al] Proposes an apparatus stash which allows the customer to make web services gave by a specific device and to hence uncover them through a REST API. [Guinard, D., et al] exhibit how particular applications can be in view of top of RESTful WSNs. [Castellani, A. P., et al, 2010] speaks to this present reality execution of a RESTful WSN. The framework/network is passed on transversely over diverse school structures and it is thought for the progression of employments and services for educators and understudies. The already expressed examination work focuses on RESTful WSNs yet don't use CoAP as application protocol. The activity of the CoRE get-together has pretty much starting late started and consequently CoAP has not yet been considered. We outlined a RESTful WSN in light of CoAP. It has twofold objective. Firstly, it depicts the huge differentiations amidst CoAP and HTTP and examines the two traditions to the extent power use and overhead. To show the upsides of CoAP, two essential examinations with the Contiki Operating System has been carried out: the first bringing into play CoAP more than 6LoWPAN and the second one exercising HTTP more than 6LoWPAN. The outcomes exhibit that the power draws on is unquestionably lower when bringing into play CoAP diverged from HTTP. In addition, we delineates the design and change of an end-to-end IP based auxiliary designing arranging a CoAP more than 6LowPAN Contiki based WSN with a HTTP over IP based application. The application allows a customer to get to WSN data particularly from a Web program (browser). The structure has been planned for keeping an eye on Greenhouse. Then again, it is work in progression and it has not yet been passed on. Accordingly, we outline how the usage of CoAP and 6LoWPAN unravels the coordination of WSNs with Web applications.

\section{THE CONSTRAINED APPLICATION PROTOCOL (COAP)}

The IETF CoRE Working Group has started the standardization activity on CoAP in late 2010. CoAP is a web transfer protocol overhauled for resource constrained frameworks regular of M2M and IoT applications. CoAP is considering a REST basic arranging in which resources are server-controlled reflections made available by an application get ready and recognized by Universal Resource Identifiers (URIs). The resources can be controlled by system for the same systems as the ones used by Hypertext Transfer Protocol (HTTP): GET, PUT, POST and DELETE. CoAP is not an outwardly weakened weight of Hypertext Transfer Protocol (HTTP). It embodies a subset of Hypertext Transfer Protocol (HTTP) functionalities which have been re-formed considering the low taking care of power and essentialness use impediments of minimal embedded devices, for instance, sensors. Besides, diverse parts and have been changed and some new functionalities have been incorporated appeal to make the tradition fitting to an applications of M2M and IoT. The essential gigantic qualification amidst HTTP and CoAP is the transport layer. HTTP relies on upon the Transmission Control Protocol (TCP). TCP's stream control approach is not fitting for LLNs and its overhead is considered too high for transitory (Short-live) trades. In like manner, TCP does not have multicast reinforce and is to a degree sensitive to movability. CoAP is in light of top of the User Datagram Protocol (UDP) and in this way has basically lower overhead and multicast support. CoAP is dealt with in two layers. The
Transaction layer levers the single message exchange between end points. The messages exchanged on this layer can be of four sorts: Confirmable (it calls for an insistence), Nonconfirmable (it doesn't need to be perceived), Acknowledgment (it perceives a Confirmable message) and Reset (it shows that a Confirmable message has been get however association is truant to be arranged). The Request/Response layer is accountable for the transmission of requests and responses for the resources control and transmission. This is the layer where the REST based correspondence happens. A REST requesting is piggybacked on a Confirmable or Non-confirmable message, while a REST response is piggybacked on the related Acknowledgment message. The twofold (dual Layer) layer procedure licenses CoAP to give steadfastness segments even without the usage of TCP as transport protocol. In reality, a Confirmable message is retransmitted brining into play a default timeout and exponential back-off between retransmissions, until the recipient sends the Acknowledgement message. Besides, it facilitates unique correspondence (Asynchronous Communication) which is a key essential for M2M and IoT applications. Right when a CoAP server gets an offer which is not prepared to handle speedily, it first perceives the reception of the message and sends back the response in a detached(offline form) from the net outline. Tokens are brought into Play for request/response fitting in Asynchronous Communication. The transaction layer moreover offers hold up to congestion control and multicast [Eggert, L]. One of the critical design goals of CoAP has been to keep the message overhead as meager as could be permitted and limit the use of irregularity. HTTP has a generally immeasurable overhead. This proposes pack break and coming about execution defilement of LLNs. CoAP make uses of a short fixed-length compact binary header of 4 bytes took after by compact binary choices. A customary offer has a total header of around 10-20 bytes. Since an advantage on CoAP server likely changes after sooner or later, the protocols facilitates a client to dependably watch the resources. This is done by system for observations: the client (the onlooker) registers itself to the resource (the subject) by strategy for a balanced GET request sent to the server. The server makes a recognition relationship between the resource and the client. At whatever points the state of the resource changes, the server exhorts each client having an observation relationship with the resource. The term of the discernment relationship is orchestrated in the midst of the enrollment/registration method [Hartke, K., et al]. Regardless of the way that CoAP is work in headway, distinctive open source executions are presently available. The two most known working systems for WSNs, Contiki and Tiny OS, have authoritatively released a CoAP draws on. Also, there are two open source executions not especially planned for WSNs: an execution in C language called libcoap [libcoap.sourceforge.net] and one in Python language called CoAPy [coapy.sourceforge.net].

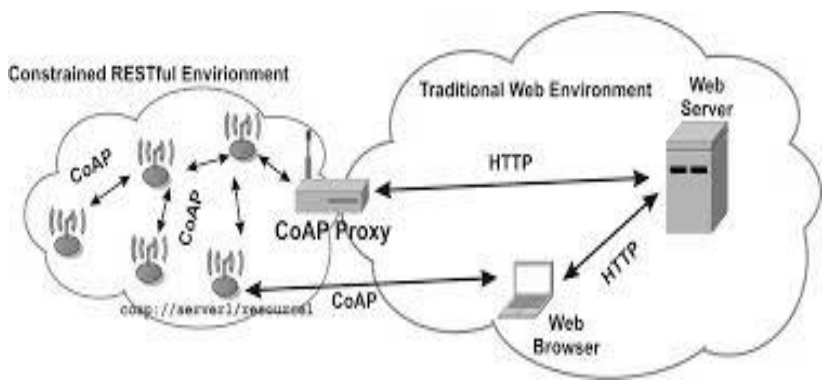

Fig 1: COAP Environment 


\section{POWER USAGE ASSESSMENT OF COAP}

UDP usage as a transport protocol and the decline of the group header measure by and large upgrade the power usage and battery lifetime in WSNs. Remembering the final objective to evaluate the execution change of CoAP appeared differently in relation to HTTP, a fundamental examination has been executed. A movement of web organization requests initially between a CoAP client/server system and a while later between a HTTP client/server structures has been carried out. The CoAP server is executed by system for a Tmote Sky sensor bit running Contiki with 6LoWPAN/RPL on the network layer and CoAP on the application layer. The CoAP bring into play Contiki starting now consolidates various highlights of the protocol, for instance, message accentuation and semantics, systems, response codes, decision fields, URIs and resource disclosure. Regardless, being worked in headway there are still discriminating functionalities missing, for instance, nonconcurrent trades, discernments and blockage/congestion control. The CoAP client is completed by running libcoap on a Linux Ubuntu machine with a USB Contiki-section which interfaces with the WSN. The HTTP server is gotten with the same Tmote Sky organize as in the CoAP server and Contiki stacked with the HTTP server instead of the CoAP server. The HTTP client is gotten by supplanting libcoap with cURL [curl.haxx.se], a charge line undertaking comprising HTTP functionalities. In both examinations the client reviews the server at general interims for twenty minutes by requesting temperature and clamminess. Right when using CoAP the sales has the going hand in hand with association: GET coap://[<mote_ip_address $>]:<$ port_number $>/$ readings, where mote_ip_address is the bit's IPv6 address, port_number is the bit's port number and readings is the benefit the client is asking (for this circumstance temperature and stickiness). Right when bringing into play HTTP the offer has the going hand in hand with course of action: GET

http://[<moteip_address>]:<port_number>/readings where the parameters have the same significance as when bringing into play CoAP.

In both CoAP and HTTP cases, the server responds by sending the sensor readings embedded into a Java Script Object Notation (JSON) report. JSON is a lightweight text based open standard for data client/server data exchange. An instance of the response's payload is the going hand in hand with: \{"sensor":" 0212:7400:0002:0202","readings":\{"hum":31,"temp":23.1\}\}, where the sensor is seen by the last four groups of its IPv6 area, hum is the humidity resource and temp is the temperature resource. CoAP moreover holds up other payload encoding rules, for instance, the for the most part used Extensible Markup Language (XML). On the other hand, the verbosity and parsing multifaceted nature of XML makes this lingo not apt for urged contraptions. Notwithstanding the way that the traditionalist/compact data representation in JSON is more fitting for WSNs, JSON does not have the flexibility of XML. Thus, there has been enormous push to make twofold/dual layer XML based representations, for instance, the Extensible XML Interchange (EXI) [Shelby, 2010]. Table 1 speaks to the outcomes of the relationship amidst CoAP and HTTP with respect to byte transferred each trade, power usage and battery lifetime. The power usage has been determined by technique for Energest, a tool prepared to gage the power use of Tmote Sky bits [Dunkels, A., et al]. The results have been taken in persevering state circumstances.

Table 1: Relationship amidst CoAP and HTTP

\begin{tabular}{|c|c|c|c|}
\hline & $\begin{array}{c}\text { Bytes per- } \\
\text { transaction }\end{array}$ & Power & Lifetime \\
\hline CoAP & 154 & $0.744 \mathrm{~mW}$ & 151 days \\
\hline$H T T P$ & 1451 & $1.333 \mathrm{~mW}$ & 84 days \\
\hline
\end{tabular}

According to aforementioned table, a HTTP transaction has different bytes ten times greater than the transaction of CoAP. This is a result of the paramount header weight executed in CoAP. Really, as aforementioned, CoAP brings into play a short fixed length compact binary header of 4 bytes and ordinary request has a total header of around 10-20 bytes. In the wake of being exemplified in the UDP, 6LoWPAN and MAC layer headers, the CoAP packet can be move into a singular MAC layout which has a size of 127 bytes. It is obvious that the higher number of bytes stirred in a HTTP transaction construes a more heightened activity of the bit's handset and CPU and therefore higher power usage $(1.33 \mathrm{~mW}$ in HTTP against $0.74 \mathrm{~mW}$ in CoAP). In both trials, the server bit was filled with 2 AA Zinc-carbon. The figures of the power usage lead to an estimation of the battery lifetime of 84 days in HTTP and 151 in CoAP. Note that the battery lifetime in both cases is absurdly short as a result of the high number of client sales made in the midst of the examination. It justifies underlining that the results showed don't altogether dissect the two protocols. The clear investigation showed is simply anticipated that would plot how the UDP tying and the header weight exhibited in CoAP upgrade the power use of WSNs.

\section{INTEGRATION OF COAP BASED WSN'S}

An IP based correspondence or communication usage and a REST based Web building outline in LLNs holds up the integration of WSNs with Internet based Web applications. The purpose of the application is to set aside a customer to get to WSN data direct from a Web program (Browser), as portrayed in Fig 2.

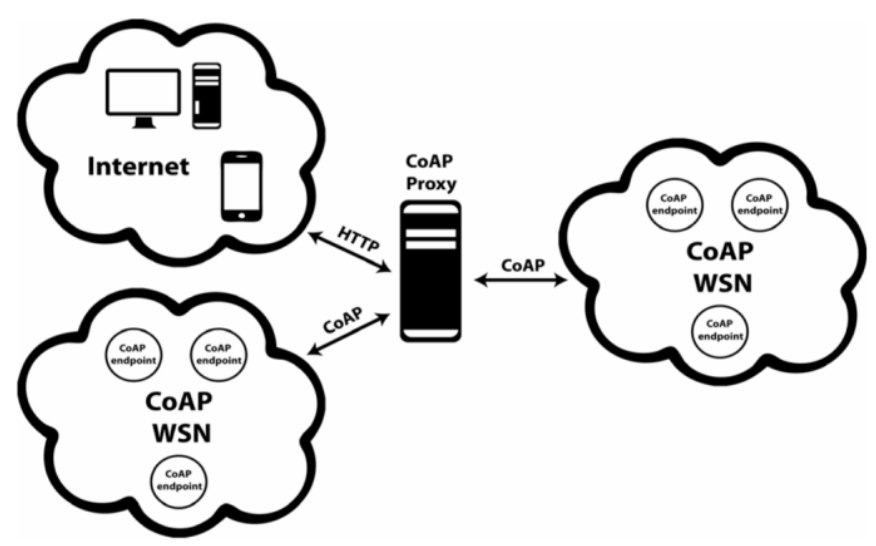

Fig (a) 


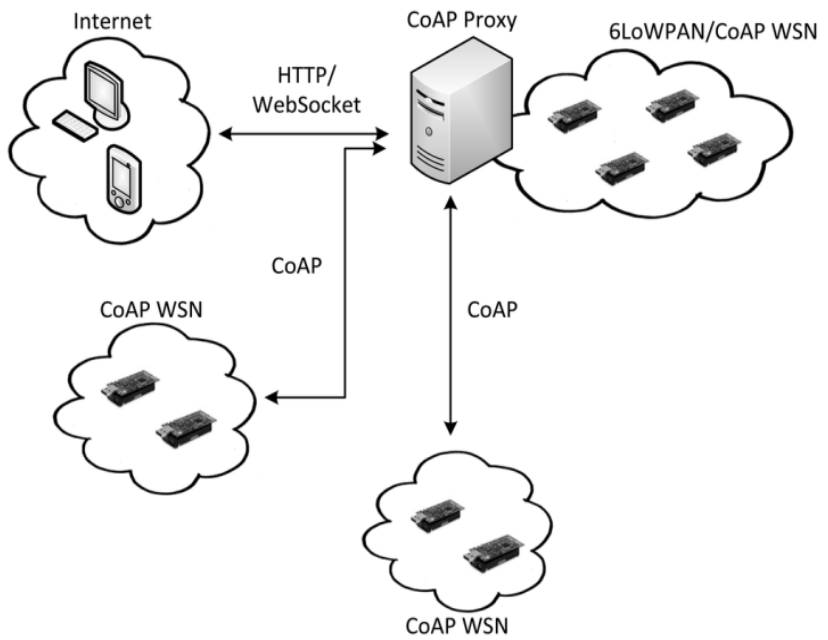

Fig (b)

Fig (a,b): Compromise amidst WSNs and the Web

The system has been expected for test observation of greenhouse. In any case, it is work in headway and it has not yet been sent.

\subsection{Designing and Constructing the \\ Gateway}

Exactly when the resources of the sensor's are uncovered by the device itself with an application protocol like CoAP, the gateway's multifaceted design is basically lessened with diverged from the case in which the sensor's resources are revealed by an application entry. To be totally straightforward, an application section needs to have full data of the functionalities of each related device. This decreases the building configuration flexibility and hampers the system adaptability. This is one of the main concerns of non IP based WSN correspondence/communication standards, for instance, ZigBee. ZigBee does not have a standard IP frameworks/networking layer which recommends that a standard web service development displaying can't be realized on top of ZigBee. Other than hampering the interoperability, the unlucky deficiency of a web service development displaying obliges the usage of utilization sections when interconnecting ZigBee WSNs to the Internet. The entryway organizing the CoAP based WSN with the HTTP based Web application comprises a Linux Ubuntu machine with a Contiki-section joined through USB port. The key building squares of the item running on the portal are portrayed in Fig 3.

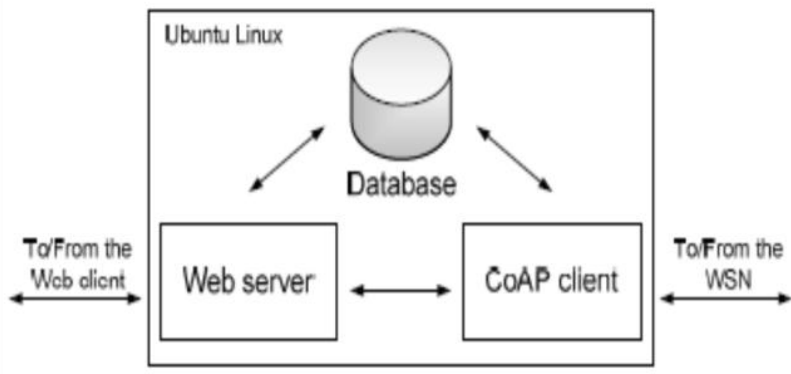

(a)

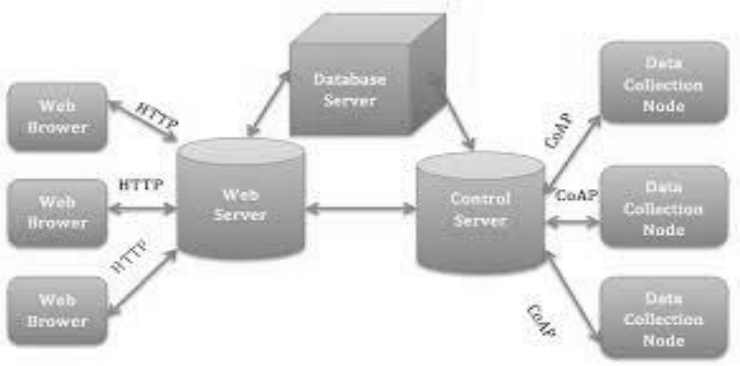

(b)

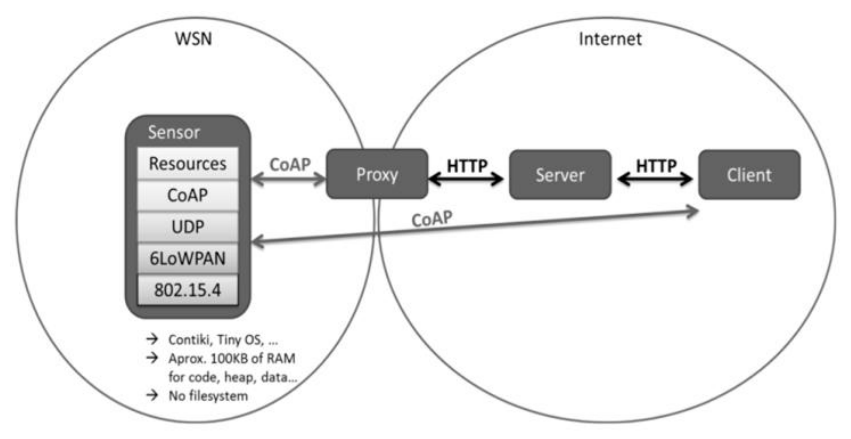

(c)

Fig 3 (a,b,c): Essential building blocks of gateway

The essential entryway's building blocks are the database, web server and the CoAP client. For straightforwardness, the first entryway model consolidates all the building blocks in the identical box. In a minute stage the application will be sent on an application server. Therefore the application basis and the data gathering handiness will stay into two extraordinary machines. This obviously diminishes the eccentrics of the entryway and upgrades the structure adaptability. The web server fuses a course of action of services which are brought into play to recoup data either from the database or particularly from the CoAP client. Right when the Web server sends the Web client chronicled data available in the database, the web server particularly gets to the database without relating with the CoAP client and sends the data back to the Web client. Exactly when the Web Server needs to send new data beginning from the WSN (upon client claim huge amounts of the WSN resources), the web server evades the database and particularly talks with the CoAP client. Since the Web application has been delivered with Google Web Toolkit (GWT), the web server at this moment is the intrinsic GWT's server called Jetty. Exactly when the application will be passed on an application server another web server will be picked. The database stores data starting from the CoAP client and makes them available to the web server. The database picked is Apache CouchDB [couchdb.apache.org]. Apache CaouchDB is a record arranged database which can be addressed and requested with MapReduce strategy making use of JavaScript. It stores JSON chronicles and gives a RESTful API. Since the CoAP client gets WSN data starting now in JSON records, the limit operation is to some degree fundamental and does not oblige any moderate data control. Nevertheless, the structure has not yet been attempted under high repeat estimation conditions and thusly the database flexibility has not yet been surveyed. If a high number of set away chronicles achieves moderate database get to, an extra data get ready layer may be obliged to decrease the data accesses stillness. The libcoap CoAP client module is responsible for talking with the WSN. In the present model the entryway WSN data exchanges are incessantly propelled 
by the CoAP client. This is a upshot of the way that Contiki does not yet reinforce discernments. We are at this time counting this value so that the WSN can all of a sudden send the CoAP client data upon resource status alteration. Once recouped the JSON data from the WSN, the CoAP client incorporate a period stamp and stores them into the database. The time stamp is obliged when outfitting the web server with undeniable data. For smoothness, the present portal utilization does reject go-between convenience amidst HTTP and CoAP. In this way there is not indulgent between the HTTP request and the CoAP offer and the other path around. In the wake of tolerating the HTTP request, the web server invokes the CoAP client with the parameters incorporated in the HTTP requests (IPv6 address and port of the bit and the advantage of diversion). This puts forwards that the passage is not utterly direct to the application and to the WSN. Proxy module prepared to do the HTTP-CoAP translation and the other route around necessities to be executed remembering the deciding objective to construct the straightforwardness of the entryway/gateway. This will similarly empower the gateway in dealing with more trapped operations, for instance, observations. For this circumstance for occurrence, a segment that unravels a HTTP enrollment (e.g. long-reviewing) needs to be translated in a CoAP discernment relationship. There is at this moment an advancing dialog in the CoRE social affair to settle on issues related to HTTP-CoAP mapping [Castellani, A., et al, Internet-Draft].

\section{CONCLUSIONS}

The fuse of WSNs with the Web has been outlined here. This is being empowered by the change of CoAP, an IETF protocol outfitting LLNs with a RESTful building configuration. CoAP bestows the identical systems for the resource control as HTTP. Additionally, CoAP supports additional functionalities normal of M2M and IoT applications, for instance, multicast, unique correspondence and enrollments. Not at all like HTTP, CoAP is taking into account top of UDP and has a littler bundle overhead. We outlined how the presentation of UDP and the packet overhead compresion fundamentally diminish the bit's vitality usage and along these lines extend the battery lifetime. In like manner it has also been depicted the design and development of an end-to-end IP based auxiliary arranging or architecture fusing a CoAP more than 6LowPAN Contiki based WSN with a HTTP over IP based application. The application facilitates a customer to get to WSN data direct from a Web program (Browser). We delineated the crucial building blocks of the section uniting the Web client with the WSN. The gateway is still in model stage and it requires the progression of proxy and discernment functionalities. The database execution needs to be striven for flexibility reason.

\section{REFERENCES}

[1] "D-Haggerty, S.", et al. sMAP - a Simple Measurement and Actuation Profile for Physical Information, the 8th ACM Conference on Embedded Networked Sensor Systems (SenSys), 2010.

[2] "Kovatsch, M., et al", Embedding Internet Technology for Home Automation. IEEE Conference on Emerging Technologies and Factory Automation (ETFA), 2010.
[3] "Mayer, S., et al", Facilitating the Integration and Interaction of Real-World Services for the Web of Things. In Proceedings of Urban Internet of Things Towards Programmable Real-time Cities (UrbanIOT), 2010.

[4] "Guinard, D., et al", A Resource Oriented Architecture for the Web of Things. In Proceedings of Internet of Things 2010 International Conference (IoT), 2010.

[5] "Castellani, A. P., et al", Architecture and Protocols for the Internet of Things: A Case Study. In Proceedings of First International Workshop on the Web of Things (WoT), 2010.

[6] "Shelby", Z. Embedded Web Services. IEEE Wireless Communications, pp. 52-57, December 2010.

[7] "Atzori, L., et al", The Internet of Things: A survey. Computer Networks, pp. 2787-2805, October 2010.

[8] "Kushalnagar", N, IPv6 over Low-Power Wireless Personal Area Networks (6LoWPANs): Overview, Assumptions, Problem Statement, and Goals. RFC 4919.

[9] "Vasseur, J. P et al", Interconnecting Smart Objects with IP: The Next Internet. Morgan Kaufmann, 2010.

[10] "Shelby, Z., \& Bormann, C.", 6LoWPAN: The Wireless Embedded Internet, Wiley, 2009.

[11] "Trifa, V., et al". Design and Implementation of a Gateway for Web-based Interaction and Management of Embedded Devices. In Proceedings of the 2nd International Workshop on Sensor Network Engineering (IWSNE), 2009.

[12] "Shelby, Z., et al", Constrained Application Protocol (CoAP). Internet-Draft. draft-ietf-core-coap-04.

[13] "Hartke, K., et al", Observing Resources in CoAP. Internet-Draft. draft-ietf-core-observe-01.

[14] "Eggert, L.", Congestion Control for the Constrained Application Protocol (CoAP). Internet-Draft. drafteggert-core-congestion-control-01.

[15 ] "Dunkels, A., et al", Demo abstract: Software-based sensor node energy estimation. In Proceedings of the Fifth ACM Conference on Networked Embedded Sensor Systems (SenSys), 2007.

[16] "Castellani, A., et al", Best Practice to map HTTP to COAP and viceversa. Internet-Draft. draft-castellanicore-http-coap-mapping-00.txt.

[17] IPSO Alliance: http://ipso-alliance.org/

[18] libcoap: C-Implementation of CoAP: http://libcoap.sourceforge.net/

[29] CoAPy: Constrained Application Protocol in Python: http://coapy.sourceforge.net/

[20] curl: http://curl.haxx.se/

[21] A Database for the Web: http://couchdb.apache.org/ 